\title{
Adolescentes con Trastorno por juego en Internet (IGD): perfiles y respuesta al tratamiento
}

\section{Adolescents with Internet Gaming Disorder (IGD): profiles and treatment response}

\author{
María Martín-Fernández*, Josep Lluís Matalí*, Sara García-Sánchez*, Marta Pardo*, María \\ Lleras*, Carmina Castellano-Tejedor**. \\ *Unidad de Conductas Adictivas. Departamento de Psiquiatría y Psicología de la Infancia y la Adolescencia. Hospital Sant Joan \\ de Déu. Barcelona (España). CIBERSAM; **Departamento de Psiquiatría. Hospital Universitari Vall d'Hebron. Barcelona \\ (España). CIBERSAM.
}

\section{Resumen}

Las demandas de tratamiento de adolescentes con problemas relacionados con el uso de videojuegos han incrementado significativamente. La mayoría de casos presentan un trastorno mental comórbido que compromete ambas patologías. El objetivo del presente estudio es describir los perfiles de adolescentes con Trastorno por Juego en Internet (IGD) según la comorbilidad y analizar la respuesta al tratamiento a los 3 y 6 meses. Se ha valorado una muestra de 86 pacientes que han consultado en la Unidad de Conductas Adictivas de un hospitalmediante los criterios del IGD, la entrevista semiestructurada K-SADS-PL para los trastornos mentales y la Impresión Clínica Global (ICG) para la evolución del tratamiento. Del total de pacientes, un $68,6 \%(\mathrm{n}=59)$ cumplían criterios para el IGD. De estos, el 45,76\% corresponderían a un perfil internalizante, presentando comorbilidades con Trastornos Afectivos (44,4\%), Trastornos de Ansiedad (44,4\%) y Trastornos de Personalidad $(11,1 \%)$. El perfil externalizante englobaría al 52,54\% de la muestra, presentando Trastorno del Comportamiento Perturbador (48,4\%), TDAH (29\%) y Trastorno del Comportamiento Perturbador no especificado (22,6\%). A diferencia de los externalizantes, los pacientes internalizantes tienen más antecedentes psiquiátricos familiares (63\%), dificultades con las relaciones sociales $(77,8 \%)$ y parecen utilizar los videojuegos preferentemente para escapar del malestar $(66,7 \%)$. A los 3 meses el perfil externalizante muestra mejorías. Se pueden discriminar dos perfiles de adolescentes con IGD en función de los trastornos comórbidos y esto puede influir en la respuesta al tratamiento. Por ello, resulta clave valorar las comorbilidades para realizar un planteamiento más eficaz del abordaje psicoterapéutico enfocado a las especificidades de cada perfil.

Palabras clave: Adolescentes; Videojuegos; IGD; Trastornos comórbidos; Tratamiento.

\begin{abstract}
Demand for treatment for problems related to the use of video games have increased significantly in adolescents. Most cases have a comorbid mental disorder that jeopardises both pathologies. The aim of this study is to describe profiles of adolescents with Internet Gaming Disorder (IGD) according to comorbidity and analyze treatment response at 3 and 6 months. A sample of 86 patients which consulted in the Addictive Behavior Unit of a hospital was assessed with diagnostic criteria for IGD, the interview K-SADS-PL for mental disorders and the Clinical Global Impression (CGI) to treatment progress. Of the initial sample, $68,6 \%(n=59)$ met diagnostic criteria for IGD. Of these, the $45,76 \%$ matched an internalizing profile, presenting comorbidity with Mood Disorders $(44,4 \%)$, Anxiety Disorders $(44,4 \%)$ and Personality Disorders $(11,1 \%)$. The externalizing profile would comprise $52,54 \%$ of the sample presenting Disruptive Behavior Disorder $(48,4 \%=$, ADHD $(29 \%)$ and Disruptive Behavior Disorders not otherwise specified $(22,6 \%)$. Unlike externalizing, the internalizing patients had a family history of psychiatric problems $(63 \%)$, difficulties in social relationships $(77,8 \%)$ and seemed to use video games preferably to escape discomfort $(66,7 \%)$. After 3 months the externalizing profile showed improvements. Comorbid disorders allow the discrimination of two IGD profiles in adolescents and these could influence treatment response. Therefore, it is important to assess comorbidities to design a more accurate intervention focused on the specificities of each profile.
\end{abstract}

Keywords: Adolescents; Video games; IGD; Comorbid disorders; Treatment. 
I nternet se ha convertido en una herramienta imprescindible en la vida cotidiana, sobre todo entre la población más joven ( Buil, Solé y García, 2015; Ko, Yen, Chen, Chen y Yen, 2012; Yau y Potenza, 2014), que lo utilizan para acceder a una gran variedad de contenidos a través de múltiples dispositivos con las principales finalidades de relacionarse, divertirse o aprender (Cho et al., 2014; Marco y Chóliz, 2014). Paralelamente, en los últimos años se ha evidenciado que el uso inadecuado o excesivo de Internet impacta negativamente sobre las funciones de la vida cotidiana, las relaciones familiares e interpersonales y la estabilidad emocional, incrementándose con todo ello los estudios sobre la adicción a Internet (Griffiths y Meredith, 2009; Ko et al., 2012; Kuss, Van Rooij, Shorter, Griffiths y Van de Mheen, 2013; Vallejos y Capa, 2010; Yau y Potenza, 2014). Dichos estudios describen que las tasas de prevalencia de Adicción a Internet en adolescentes oscilan entre el 1,7\% y el 10\% (Kuss,et al., 2013; Matalí-Costa, Serrano-Troncoso, Pardo, Villar y San, 2014; Petry et al., 2014; Yau y Potenza, 2014). Una revisión realizada por Ferguson y colaboradores concluye que las estimaciones sobre el uso verdaderamente problemático parecen ser menores a las descritas en algunos trabajos, situándose alrededor del 3,1\% (Ferguson, Coulson y Barnett, 2011; Van Rooij, Kuss, Griffiths, Shorter, Schoenmakers y Van de Mheen, 2014) o entre el 2\% y el $5 \%$ según otros autores (Kuss et al., 2013; Rehbein, Kliem, Baier, Mößle y Petry, 2015). En relación a la edad, el consenso es mayor, siendo la población adolescente la que presenta mayores problemáticas en cuanto al uso y/o abuso de Internet (Carbonell, 2014; Buil, Solé y García, 2015; Ferguson et al., 2011; Kuss et al., 2013). En el uso de los videojuegos, se observan diferencias de género, siendo los hombres más propensos a jugar que las mujeres (Fernández-Villa et al., 2015; Király, Nagygyörgy, Griffiths y Demetrovics, 2014; Kuss et al., 2013; Lemos, De Abreu y Sougey, 2014).

El Manual Diagnóstico y Estadístico de los Trastornos mentales, en su última revisión (DSM5), señala que la Adicción a Internet no tiene entidad suficiente como para ser catalogada como trastorno pero propone la existencia del Trastorno por Juego en Internet (Internet Gaming Disorder, en adelante IGD por sus siglas en inglés) indicando que requiere de estudios posteriores (American Psychiatric Association, 2013; Carbonell, 2014; Petry y O'Brien, 2013). Dicha situación ha suscitado diversas investigaciones (Lopez-Fernandez, Honrubia-Serrano, Baguley y Griffiths, 2014) proporcionando evidencia suficiente de que usar los videojuegos de manera desmedida es una actividad potencialmente problemática. En relación al tipo de videojuegos, los Massively Multiplayer Online Role-Playing Games (MMORPG) son los que generan más repercusiones y parecen tener una capacidad adictiva mayor, ya que estos se caracterizan por ofrecer una actividad sin final establecido así como numerosas posibilidades de acción, donde los jugadores crean su avatar y progresan en el juego creando clanes con otros jugadores. Al acabar cada misión, el jugador obtiene una recompensa en forma de habilidad o de información que le sitúa en una nueva posición de cara a continuar con el juego (Carbonell, 2014). Todo ello, ocasiona dificultades para poder dejar de jugar y una pérdida de control en relación al tiempo de uso (Griffiths y Meredith, 2009; Marco y Chóliz, 2014).

Los pacientes con IGD presentan unas elevadas tasas de comorbilidad con otros trastornos mentales (Yen, Ko, Yen, Wu y Yang, 2007), siendo los más frecuentes los Trastornos del Estado de Ánimo, el Trastorno de Ansiedad Generalizada, el Trastorno de Pánico, la Fobia Social, el Trastorno Obsesivo Compulsivo, el Trastorno por Uso de Sustancias, el Trastorno por Déficit de Atención e Hiperactividad, Trastornos de conducta, Trastornos de Personalidad y Trastornos Psicóticos (Echeburúa y Corral Gargallo, 2010; Ko et al., 2012; Marco y Chóliz, 2014; Van Rooij et al., 2014; Yau y Potenza, 2014; Yen et al., 2007). La controversia o dificultad en el diagnóstico diferencial reside en si el uso desadaptativo de los videojuegos es un trastorno en sí mismo o se trata de un síntoma de otro trastorno mental, dada la alta frecuencia de comorbilidad existente (Echeburúa y Corral Gargallo, 2010; Yen et al., 2007), tal como ocurre con otras adicciones (Király et al., 2014; Matalí-Costa et al.,2014), resultando imprescindible una buena evaluación para poder entender cómo se ha instaurado el cuadro desadaptativo y para posteriormente determinar el tipo de intervención que requerirá el paciente (Yen et al., 2007).

En los últimos años se están incrementando el número de demandas de tratamiento por presentar problemas con el uso de los videojuegos entre población menor de edad (Matalí-Costa et al., 2014). En contraste, existe poca evidencia sobre el tipo de abordaje para el IGD (Griffiths y Meredith, 2009; Marco y Chóliz, 2014). Según la literatura, el tratamiento cognitivo-conductual es el más eficaz para la identificación de los problemas relacionados con los videojuegos, su modificación y sustitución por otras pautas más adaptativas (Echeburúa y Corral Gargallo, 2010; Petry et al., 2014; Young, 2007). Algunas de las intervenciones que se habían planteado para la Adicción a Internet pueden resultar de gran utilidad para el tratamiento del actual IGD. En este sentido, existe un consenso en todos los estudios, que determina como principal objetivo del tratamiento el uso controlado y adaptativo de los videojuegos (Arias Rodríguez, Gallego Pañeda, Rodríguez Nistal, y Del Pozo López, 2012; Echeburúa y Corral Gargallo, 2010; King y Delfabbro, 2014; Yau y Potenza, 2014).

La literatura demuestra que existen diferentes perfiles de uso de Internet y en consecuencia, la respuesta al tratamiento podría resultar condicionada (Matalí-Costa et al., 2014). En el presente trabajo, se plantea que con las comorbilidades que acompaña al IGD se podrían formar unos perfiles en los que la respuesta al tratamiento sería diferente. Para comprobarlo, se diseñó un estudio con dos 
objetivos principales. En un primer momento, establecer perfiles en una muestra clínica de pacientes adolescentes con diagnóstico de IGD en función de los trastornos comórbidos internalizantes o externalizantes y, describir las características clínicas y el patrón de uso de los videojuegos en ambos perfiles. A continuación, analizar la respuesta al tratamiento a los 3 y 6 meses de estos adolescentes en función del perfil, valorando el tipo y la intensidad de la intervención realizada.

\section{Método}

El diseño del presente trabajo es de tipo transversal.

\section{Muestra}

Se analizaron los datos de un total de 86 pacientes ambulatorios remitidos a la Unidad de Conductas Adictivas del Servicio de Psiquiatría y Psicología de la Infancia y la Adolescencia, por presentar problemática con Internet o los videojuegos durante el período de 2009 a 2015.

Los criterios de inclusión de la muestra fueron: ser menor de 18 años de edad en el momento de la primera visita, presentar un uso desadaptativo de Internet o de videojuegos y cumplir con los criterios de IGD del DSM- 5 , valorado retrospectivamente. Se excluyeron 27 pacientes por no cumplir los criterios de inclusión. Así, la muestra final del estudio estuvo constituida por 59 adolescentes.

\section{Aspectos éticos}

Para llevar a cabo la presente investigación, se obtuvo la aprobación del Comité de Ética hospitalario, considerando la normativa ética interna, así como la de la Asociación Médica Mundial y la Declaración de Helsinki de 1995 y sus posteriores enmiendas. Todos los participantes otorgaron su consentimiento verbal tras recibir la información relativa al trabajo y sus objetivos.

\section{Procedimiento}

Esta investigación se llevó a cabo íntegramente en la Unidad de Conductas Adictivas perteneciente al Servicio de Psiquiatría y Psicología de la Infancia y la Adolescencia. A partir de las historias clínicas hospitalarias se revisaron los datos socio-demográficos, los datos clínicos, los relativos al patrón de juego, al tratamiento recibido y a su evolución a los 3 y 6 meses.

De la información obtenida en la primera sesión de evaluación, en la que se realizaba una entrevista clínica con el adolescente, se valoraba la posible problemática con Internet y/o los videojuegos y la presencia de IGD, (según criterios DSM-5) y la posibilidad de presentar o no otro trastorno mental asociado. A todos los pacientes que cumplían los criterios de inclusión se registraban las siguientes variables:

- Datos socio-demográficos: género, edad y curso académico actual.
- Variables clínicas: para determinar el diagnóstico de IGD se utilizaron los criterios propuestos en el DSM-5, y para evaluar la existencia de otros trastornos mentales comórbidos se utilizó la entrevista semi-estructurada Kiddie-sads-present $\mathcal{E}$ Lifetime (Kaufman el al., 1997; Ulloa et al., 2006) creada para evaluar la psicopatología en niños y adolescentes de 6 a 17 años y que se fundamenta en los criterios del DSM-IV-TR. Los coeficientes de fiabilidad de la versión española de la escala van de 0.76 para el trastorno depresivo a valores próximos a 1 para el trastorno disocial. Además se evaluó si habían tenido tratamientos previos, más de un diagnóstico y la existencia de antecedentes familiares psiquiátricos.

- Variables psicosociales: se recogió la existencia de problemas con el grupo de apoyo primario (familia) y con el grupo de amigos, la existencia de acoso escolar o bullying, la posible pérdida de contacto (previa a la problemática) con el grupo de amigos por cambiar de escuela o de domicilio, cambios en el rendimiento escolar y consumo de drogas.

- Variables sobre el patrón de juego: se registró el horario de juego, la preferencia por el juego de tarde o nocturno, el tipo de aplicaciones que utilizan (juegos online, juego MMORPG y/o chat), el principal motivo por el que usan los videojuegos y la queja que presenta delante de la retirada de éste (de aburrimiento o incapacidad).

- Tratamiento y evolución: se recogió la información relacionada con la intensidad de la intervención (consultas externas, hospitalización parcial o total), y el tipo de intervención (psicoterapéutica, farmacológica o combinada). La evolución a los tres y a los seis meses se valoró con la escala de Impresión Clínica Global (CGI-I). El rango de puntuaciones de esta escala oscila entre el 0 y el 7; del 1 al 3 el paciente muestra mejorías, el 4 indica que se mantiene la problemática, y del 5 al 7 indica un empeoramiento de la sintomatología.

Todos los participantes incluidos en el estudio fueron tratados de manera individualizada desde un enfoque psicoterapéutico siguiendo el modelo de las adicciones conductuales propuesto por Echeburúa (Echeburúa y Corral Gargallo, 2010) y en los casos en que resultó necesario, con tratamiento farmacológico.

Para realizar los perfiles, se utilizaron los criterios seguidos en el estudio de Matalí-Costa y colaboradores (Matalí-Costa et al., 2014), en el cual se clasifica la muestra de estudio en dos grupos diferenciados según la clasificación de Achenbach de los trastornos mentales, internalizante y externalizante (Achenbach y Edelbrock, 1984; Matalí-Costa et al., 2014). De tal manera, los perfiles se realizaron en función del diagnóstico comórbido que presentaban los adolescentes. Por un lado, el grupo con perfil exter- 
nalizante, aquel integrado por adolescentes que presentan alteraciones en el control de comportamientos tales como agresión, impulsividad, negativismo e hiperactividad. Los adolescentes de dicho perfil presentan Trastornos de Comportamiento Perturbador, TDAH y Trastorno Comportamiento Perturbador no especificado. Por otro lado, el grupo de internalizantes manifiestan los problemas o dificultades mediante la inhibición, inquietud, evitación o la timidez. En este caso los sujetos de la muestra presentan Trastorno Depresivo, Ansiedad, Trastornos de Personalidad (Clúster C); (Achenbach y Edelbrock, 1984).

\section{Análisis estadístico}

Se realizaron análisis descriptivos utilizando frecuencias y porcentajes para las variables categóricas y la media y la desviación estándar para las variables cuantitativas. Para evaluar las diferencias entre los perfiles propuestos, se ha realizado la prueba de Chi-cuadrado, la prueba de Fisher y la prueba t de Student según procedía. Para realizar los análisis estadísticos se utilizó el SPSS 18.8 (IBM Corp). La significación de todas las pruebas se ha considerado con un nivel de probabilidad del $5 \%$ o inferior, indicando siempre la significación exacta que ofrecía el SPSS.

\section{Resultados}

De los 86 pacientes que han asistido a la Unidad de Conductas Adictivas por presentar problemas con Internet o los videojuegos, 59 cumplen criterios para el IGD (DSM-5). Las edades de estos 59 casos, se comprenden entre los 12

Tabla 1. Variables del perfil clínico.

\begin{tabular}{|c|c|c|c|c|c|}
\hline & \multicolumn{2}{|c|}{ Internalizante $(n=27)$} & \multicolumn{2}{|c|}{ Externalizante $(n=31)$} & \multirow[b]{2}{*}{$\mathbf{p}$} \\
\hline & $\%$ & $\mathbf{n}$ & $\%$ & $\mathbf{n}$ & \\
\hline \multicolumn{6}{|l|}{ Diagnóstico } \\
\hline Trastorno Afectivo & 44,4 & 12 & 0 & 0 & \multirow{5}{*}{$p<, 001$} \\
\hline Trastorno Comportamiento Perturbador & 0 & 0 & 48,4 & 15 & \\
\hline Trastorno Ansiedad & 44,4 & 12 & 0 & 0 & \\
\hline Trastorno Personalidad & 11,1 & 3 & 0 & 0 & \\
\hline Trastorno Comportamiento Perturbador n.e & 0 & 0 & 22,6 & 7 & \\
\hline \multicolumn{6}{|l|}{ Más de un diagnóstico } \\
\hline Sí & 81,5 & 22 & 19,4 & 6 & $p<, 001$ \\
\hline \multicolumn{6}{|l|}{ Tratamientos anteriores } \\
\hline Sí & 85,2 & 23 & 48,4 & 15 & \multirow{2}{*}{$p=, 003$} \\
\hline No & 14,8 & 4 & 51,6 & 16 & \\
\hline \multicolumn{6}{|l|}{ Antecedentes familiares psiquiátricos } \\
\hline Sí & 63 & 17 & 12,9 & 4 & \multirow{2}{*}{$p<, 001$} \\
\hline No & 37 & 10 & 83,9 & 26 & \\
\hline \multicolumn{6}{|l|}{ Acoso / Bullying / Pérdidas previas } \\
\hline Sí & 77,8 & 21 & 45,2 & 14 & \multirow{2}{*}{$p=, 011$} \\
\hline No & 22,2 & 6 & 54,8 & 17 & \\
\hline \multicolumn{6}{|l|}{ Nivel escolar } \\
\hline Conservado & 11,1 & 3 & 22,6 & 7 & \multirow{2}{*}{ p>,05 } \\
\hline Fracaso & 22,2 & 6 & 9,7 & 3 & \\
\hline \multicolumn{6}{|l|}{ Distocia familiar } \\
\hline Sí & 59,3 & 16 & 80,6 & 25 & \multirow{2}{*}{$p=, 074$} \\
\hline No & 40,7 & 11 & 19,4 & 6 & \\
\hline \multicolumn{5}{|l|}{ Consumo de drogas } & \\
\hline Sí & 11,1 & 3 & 12,9 & 4 & \multirow{2}{*}{$p=, 834$} \\
\hline No & 88,9 & 24 & 87,1 & 27 & \\
\hline
\end{tabular}


Tabla 2. Variables del patrón de uso de los videojuegos.

\begin{tabular}{|c|c|c|c|c|c|}
\hline & \multicolumn{2}{|c|}{ Internalizante $(n=27)$} & \multicolumn{2}{|c|}{ Externalizante $(n=31)$} & \multirow[b]{2}{*}{$\mathbf{p}$} \\
\hline & $\%$ & $\mathbf{n}$ & $\%$ & $\mathbf{n}$ & \\
\hline \multicolumn{6}{|c|}{ Grupo de amigos conservado } \\
\hline Sí & 18,5 & 5 & 61,3 & 19 & \\
\hline No & 81,5 & 22 & 38,7 & 12 & $p=, 001$ \\
\hline \multicolumn{6}{|c|}{ Quejas sin Internet } \\
\hline Aburrimiento & 25,9 & 7 & 61,3 & 19 & \\
\hline Incapacidad & 59,3 & 16 & 16,1 & 5 & $p=, 003$ \\
\hline Ambas & 14,8 & 4 & 22,6 & 7 & \\
\hline \multicolumn{6}{|l|}{ Horario de juego } \\
\hline Tardes & 25,9 & 7 & 71 & 22 & \\
\hline Tardes / Noches & 74,1 & 20 & 29 & 9 & $p<, 001$ \\
\hline \multicolumn{6}{|l|}{ Tipo de aplicación } \\
\hline Juego Online & 0 & 0 & 38,7 & 12 & \\
\hline MMORPG & 81,5 & 22 & 25,8 & 8 & \\
\hline Chat y MMORPG & 11,1 & 3 & 29 & 9 & \\
\hline Chat & 3,7 & 1 & 6,5 & 2 & \\
\hline Juego Offline & 3,7 & 1 & 0 & 0 & $p<, 001$ \\
\hline \multicolumn{6}{|c|}{ Motivo principal de juego } \\
\hline Lúdico & 3,7 & 1 & 80,6 & 25 & \\
\hline Refugio & 66,7 & 18 & 6,5 & 2 & \\
\hline Ambos & 29,6 & 8 & 6,5 & 2 & \\
\hline Desconocido & 18,5 & 5 & 9,7 & 3 & $p<, 001$ \\
\hline
\end{tabular}

Nota: $M M O R P G=$ Massively Multiplayer Online Role-Playing Games.

y los 17 años de edad $(\mathrm{M}=14,83 ; \mathrm{SD}=1,45)$ y el sexo es masculino en el $96,6 \%$ de los casos $(n=57)$.

Los 59 sujetos diagnosticados se agruparon en el grupo internalizante o en el grupo externalizante, en función del trastorno comórbido que presentaban. El perfil internalizante está constituido por el $45,76 \%(n=27)$ de la muestra con una edad media de 15,19 años de edad $(\mathrm{SD}=1,62)$ y el perfil externalizante lo compone el 52,54\% ( $\mathrm{n}=31)$ de la muestra con una edad media de 14,48 años $(\mathrm{SD}=1,23)$. Los principales diagnósticos que corresponden al perfil internalizante son: Trastornos Afectivos (44,4\%; $\mathrm{n}=12)$, Trastornos de Ansiedad (44,4\%; n = 12) y en menor medida, Trastornos de Personalidad del Clúster C (11,1\%; n =3). En el grupo externalizante, los diagnósticos observados han sido: Trastorno de Comportamiento Perturbador $(48,4 \% ; \mathrm{n}=15)$, TDAH $(29 \% ; \mathrm{n}=9)$ y Trastorno Adaptativo $(22,6 \% ; \mathrm{n}=7)$. No se ha observado diferencias estadísticamente significativas en cuanto al sexo ni la edad de los adolescentes entre los dos grupos.

En las Tablas 1 y 2 se muestra una comparación entre los dos perfiles de sujetos, tanto de las variables clínicas como de las relacionadas con el patrón de uso de los videojuegos.

En relación con las variables clínicas, se observa que los adolescentes internalizantes han recibido más de un diag- nóstico diferente en un $81,5 \%$ de los casos y tratamientos anteriores en un $85,2 \%$, a diferencia de lo que sucede en el caso de los externalizantes, con un $19,4 \%$ y un $48,4 \%$ respectivamente. También se muestra que en el grupo internalizante un $63 \%$ de casos tiene antecedentes psiquiátricos familiares frente al 12,9\% de los externalizantes. Así mismo, a nivel social, un $77,8 \%$ de los internalizantes reportan una pérdida previa de contacto con el grupo de amigos o haber sufrido bullying en algún momento, frente a un $45,2 \%$ de los externalizantes. Las variables de rendimiento académico, distocia familiar y consumo de drogas, no muestran diferencias estadísticamente significativas entre los grupos.

En cuanto a las variables relacionadas con el uso de los videojuegos, los sujetos del grupo externalizante reportan en un $80,6 \%$ que la principal motivación para utilizarlos es recreativa. En cambio los internalizantes, en el 66,7\% de los casos, reportan un uso para refugiarse o evitar un malestar. Frente a la retirada del juego o a la imposibilidad de poder jugar, un 59,3\% de adolescentes internalizantes expresan sentirse incapaces de afrontar la situación, a diferencia de los externalizantes que reportan sentirse aburridos en el $61,3 \%$ de los casos. Por otro lado, se observa que un $74,1 \%$ de los sujetos internalizantes muestran una preferencia por jugar en horario nocturno. En relación a 
las aplicaciones más frecuentemente utilizadas, el grupo externalizante expresa una mayor variedad de preferencias utilizando tanto los juegos online $(38,7 \%)$, los MMORPG $(25,8 \%)$, los MMORPG junto con las redes sociales $(29 \%)$ y únicamente las redes sociales $(6,5 \%)$. En cambio, el grupo internalizante muestra una clara propensión a jugar principalmente a los MMORPG en un $81,5 \%$ de los casos. Un $11,1 \%$ a parte de utilizar los MMORPG utiliza también las redes sociales, un 3,7\% utiliza únicamente las redes sociales y un 3,7\% utiliza juegos sin Internet. La mayor parte de los adolescentes del grupo internalizante no conserva las amistades $(81,5 \%)$.

Con respecto a abordaje terapéutico, en la tabla 3 se muestra la comparación entre el perfil internalizante y el perfil externalizante, en relación a la intensidad del tratamiento. Es decir, si la intervención se ha realizado en consultas externas o ha requerido de hospitalización parcial o total. Asimismo, también se compara el tipo de abordaje realizado (psicoterapéutico, farmacológico o combinado), y la evaluación a los 3 y a los 6 meses.

En relación a las variables que valoran la intensidad del tratamiento, se observan diferencias estadísticamente significativas en cuanto a la hospitalización total, con un $25,9 \%(\mathrm{n}=7)$ del perfil internalizante frente al 3,2\% $(\mathrm{n}=$ 1) de los externalizantes. En cambio, estas diferencias no se observan en cuanto al tratamiento en consultas externes o en régimen de hospitalización parcial. Tampoco se observan diferencias estadísticamente significativas entre grupos en cuanto al tipo de abordaje propuesto.

En relación a la evolución del trastorno durante el tratamiento a los 3 meses, un $63 \%$ de los sujetos internalizantes mantienen la sintomatología, un 14,8\% la empeoran y un $18,5 \%$ comienzan a mostrar mejorías. En el caso de los externalizantes, un $41,9 \%$ mantienen la sintomatología, un $3,1 \%$ empeoran, y un $48,4 \%$ muestran mejorías. A los seis meses, no se encuentran diferencias estadísticamente significativas entre los grupos.

\section{Discusión}

Este estudio compara el perfil de los adolescentes con diagnóstico de IGD en función de la comorbilidad del trastorno mental que presentan (internalizante vs. externalizante) resaltando la importancia del tipo de comorbilidad

Tabla 3. Variables del abordaje terapéutico.

\begin{tabular}{|c|c|c|c|c|c|}
\hline & \multicolumn{2}{|c|}{ Internalizante $(n=27)$} & \multicolumn{2}{|c|}{ Externalizante $(n=31)$} & \multirow[b]{2}{*}{$\mathbf{p}$} \\
\hline & $\%$ & $\mathrm{n}$ & $\%$ & $n$ & \\
\hline \multicolumn{6}{|c|}{ Consultas Externas } \\
\hline Sí & 100 & 27 & 100 & 27 & \multirow{2}{*}{$p>05$} \\
\hline No & 0 & 0 & 0 & 0 & \\
\hline$\overline{\text { Sí }}$ & 25,9 & 7 & 3,2 & 1 & \multirow{2}{*}{$p=, 012$} \\
\hline No & 74,1 & 20 & 96,8 & 30 & \\
\hline \multicolumn{6}{|c|}{ Hospitalización parcial } \\
\hline Sí & 14,8 & 4 & 3,2 & 1 & \multirow{2}{*}{$p=, 117$} \\
\hline No & 85,2 & 23 & 96,8 & 30 & \\
\hline Psicoterapéutica & 48,1 & 13 & 64,5 & 20 & \multirow{3}{*}{$p=, 209$} \\
\hline Farmacológica & 0 & 0 & 0 & 0 & \\
\hline Combinada & 51,9 & 14 & 35,5 & 11 & \\
\hline \multicolumn{6}{|c|}{ Evolución a los 3 meses } \\
\hline Igual & 63 & 17 & 41,9 & 13 & \multirow{4}{*}{$p=, 027$} \\
\hline Peor & 14,8 & 4 & 3,2 & 1 & \\
\hline Mejor & 18,5 & 5 & 48,4 & 15 & \\
\hline Desconocido & 3,7 & 1 & 6,5 & 2 & \\
\hline \multicolumn{6}{|c|}{ Evolución a los 6 meses } \\
\hline Igual & 29,6 & 8 & 22,6 & 7 & \multirow{3}{*}{$p=, 562$} \\
\hline Peor & 11,1 & 3 & 6,5 & 2 & \\
\hline Desconocido & 14,8 & 4 & 12,9 & 4 & \\
\hline
\end{tabular}


tanto en los motivos de uso de los videojuegos, como en la presentación clínica y en su evolución. Así, el objetivo del presente estudio resulta especialmente relevante debido a la escasez de trabajos existentes en este ámbito y a las implicaciones clínicas y psicoterapéuticas que pueden derivar de una correcta evaluación de estos adolescentes.

En primer lugar cabe destacar que en nuestro estudio todos los casos presentan comorbilidades con el diagnóstico de IGD. Resultados similares han sido hallados en estudios anteriores, donde la comorbilidad sin estar en el total de los pacientes, si es elevada (Chin, Shin y Kim, 2006; Ferguson et al., 2011). Las comorbilidades más prevalentes han sido la depresión, la ansiedad social, el TDAH y las conductas agresivas (Fernández-Villa et al., 2015; Ko et al., 2012).

Centrándonos en las motivaciones de uso de los videojuegos, se observan diferencias entre los perfiles en función del grupo comórbido (internalizante vs. externalizante). Por un lado, los externalizantes tienden a jugar con una finalidad más recreativa a pesar de las consecuencias familiares e interpersonales asociadas (Ko et al., 2012), resultando el componente desafiante característico de estos adolescentes uno de los principales motores de perpetuación de la problemática con el uso de los videojuegos (Holtz y Appel, 2011). Por otro lado, los internalizantes utilizan los MMORPG, aplicación descrita en la literatura potencialmente adictiva (Carbonell, 2014), como una estrategia de afrontamiento para reducir el malestar provocado por los problemas que presentan en la esfera interpersonal (Carbonell, 2014; Fernández-Villa et al., 2015; Ko et al., 2012; Matalí-Costa et al., 2014); evidenciándose que los MMORPG son utilizados a su vez como una herramienta de socialización (Carbonell, 2014; Király et al., 2014). El estudio y entendimiento de las motivaciones de uso de los videojuegos es un aspecto clave para el diseño de la intervención terapéutica (Matalí-Costa et al., 2014) ya que la eficacia del correcto diseño depende de las especificad de cada caso.

El perfil comórbido internalizante, como se ha comentado anteriormente, suele utilizar el juego como una manera de paliar el malestar derivado por los problemas en las relaciones con sus iguales presentando con frecuencia elevados niveles de soledad (Fernández-Villa et al., 2015; Matalí-Costa et al., 2014), que derivan en ocasiones en una reclusión física en el domicilio, hecho que deteriora a su vez el funcionamiento socio-familiar (Teo, 2013). Este factor se ha descrito como principal factor de riesgo para el desarrollo del IGD (Ferguson et al., 2011; Fernández-Villa et al., 2015; Marco y Chóliz, 2014; Matalí-Costa et al., 2014).

En ambos perfiles se observan altas tasas de problemática en la esfera familiar y problemática en la esfera escolar. La literatura indica que la presencia de distocia familiar es un claro factor de riesgo para presentar un uso problemático con los videojuegos (Echeburúa-Odriozola, 2012;
Fernández-Villa et al., 2015) y el deterioro del rendimiento académico; que es una de las principales señales de alarma que evidencian a las familias la existencia de la problemática con los videojuegos (Echeburúa y Corral Gargallo, 2010), convirtiéndose las dos problemáticas en ámbitos de intervención.

En relación al horario de juego, se observa una preferencia del perfil internalizante por jugar en horario nocturno. Como apunta la literatura, la inversión de los estados de sueño-vigilia se han descrito como una de las señales de alarma que denotan un patrón de uso problemático (Echeburúa y Corral Gargallo, 2010; Fernández-Villa et al. 2015), y que ocasionan numerosas interferencias en las actividades de la vida cotidiana del menor (Griffiths y Meredith, 2009; Marco y Chóliz, 2014).

Por otro lado, en referencia a las propuestas de abordaje terapéutico, los resultados de este estudio apoyan la teoría que hay que adaptar dicho tratamiento en función del perfil. Los pacientes con un perfil externalizante responden mejor en menor número de visitas, y a los tres meses ya se empiezan a observar cambios (Matalí-Costa et al., 2014). En la mayoría de casos el tratamiento es ambulatorio y principalmente psicoterapéutico, dirigido a modificar los patrones de juego desadaptativos (Echeburúa y Corral Gargallo, 2010; King y Delfabbro, 2014). Esto se puede explicar, puesto que los sujetos de este perfil responden al modelo propuesto para explicar los IGDs, en el cual, la conducta problema se entiende como un Trastorno de Control de Impulsos (Young, 2007). Por tanto, encontramos que responden satisfactoriamente al establecimiento de límites con ayuda de los familiares, buscando la abstinencia parcial para conseguir un reaprendizaje del uso de los videojuegos (Echeburúa y Corral Gargallo, 2010; Grant, Potenza, Weinstein y Gorelick, 2010; King y Delfabbro, 2014). Por el contrario, los pacientes con un perfil internalizante, presentan una evolución más lenta o tórpida, requiriendo con mayor frecuencia un abordaje combinado y comprehensivo (King y Delfabbro, 2014), priorizando el abordaje de la comorbilidad como a las dificultades con las relaciones sociales (King y Delfabbro, 2014; Matalí-Costa et al., 2014).

Este estudio no está exento de limitaciones. La primera de ellas concierne al reducido tamaño de la muestra que podría estar limitando la potencia estadística y los resultados obtenidos. Asimismo, se trata de una muestra clínica por lo que los resultados no pueden generalizarse a población general. Por otro lado, el establecimiento de los criterios del IGD es reciente y todavía son escasos los estudios que lo aborden y validen empíricamente. No obstante, a la luz de los resultados obtenidos, parecen necesarios más trabajos empíricos que contrasten su pertinencia y que exploren los diferentes perfiles de pacientes con IGD de cara a un planteamiento psicoterapéutico más eficaz y específico. 
En el presente estudio se ha propuesto el establecimiento de diversos perfiles de adolescentes con diagnóstico de IGD en función del Trastorno mental comórbido (internalizante / externalizante). De los resultados obtenidos en el estudio se pueden extraer las siguientes conclusiones. La evaluación del trastorno mental comórbido que acompaña al diagnóstico de IGD ayuda en la comprensión de los motivos principales por los que el adolescente utiliza los videojuegos y en ocasiones llega a establecer unos patrones de uso desadaptativos. Se observa que la presencia de los problemas en las relaciones interpersonales o de los déficits en las habilidades sociales, así como la soledad, resultan un importante motor para que los adolescentes se recluyan en los videojuegos. Concretamente, los juegos más usados en estos casos son los MMORPG, utilizados tanto como una herramienta de afrontamiento del malestar como una herramienta de socialización principalmente para aquellos adolescentes con diagnósticos comórbidos internalizantes.

Así pues, resulta relevante definir los perfiles de los adolescentes con IGD ya que nos ayuda a guiar el plan de intervención y a identificar a aquellas personas en situación de riesgo para poder contribuir en la prevención. Se debe tener presente que la respuesta al tratamiento resulta diferente en función del perfil del adolescente y de esta manera, el abordaje deberá ser planteado de manera diferente, teniendo en cuenta que los adolescentes con IGD del perfil Internalizante son pacientes más complejos que generalmente requerirán de diferentes profesionales y recursos asistenciales para su abordaje psicoterapéutico. Los problemas relacionados con el uso de los videojuegos encubren generalmente otras dificultades de la esfera interpersonal, emocional y/o cognitiva que requieren de intervenciones más extensas, dirigidas tanto a los aspectos individuales, familiares como sociales.

\section{Conflicto de intereses}

Los autores declaran no tener ningún conflicto de intereses.

\section{Referencias}

Achenbach, T. M. y Edelbrock, C. S. (1984). Psychopathology of childhood. Annual Review of Psychology, 35, 227256. doi:10.1146/annurev.psych.35.1.227.

American Psychiatric Association (2013). Diagnostic and Statistical Manual of Mental Disorders (5th edition). Washington, DC: American Psychiatric Association.

Arias Rodríguez, O., Gallego Pañeda, V., Rodríguez Nistal, M. J. y Del Pozo López, M. Á. (2012). Adicción a las nuevas tecnologías. Psicología de las Adicciones, 1, 2-6.

Buil, P., Solé, M.J. y García, P. (2015). La regulación publicitaria de los juegos de azar online en España. Una reflexión sobre la protección del menor. Adicciones, 27, 198 - 204.

Carbonell, X. (2014). La adicción a los videojuegos en el DSM-5. Adicciones, 26, 91-95.

Chin, B., Shin, D. y Kim, J. H. (2006). Psychiatric Comorbidity Assessed in Korean Children and Adolescents who screen Positive for Internet Addiction. Journal Clinical Psychiatry, 67, 821-826.

Cho, H., Kwon, M., Choi, J.H., Lee, S.K., Choi, J. S., Choi, S.W. y Kim, D.J. (2014). Development of the Internet addiction scale based on the Internet Gaming Disorder criteria suggested in DSM-5. Addictive Behaviors, 39, 1361-1366. doi:10.1016/j.addbeh.2014.01.020.

Echeburúa, E. y Corral Gargallo, P. (2010). Adicción a las nuevas tecnologías y a las redes sociales en jóvenes: un nuevo reto. Adicciones, 22, 91-96.

Echeburúa Odriozola, E. (2012). Factores de riesgo y factores de protección en la adicción a las nuevas tecnologías y redes sociales en jóvenes y adolescentes. Revista Española de Drogodependencias, 37, 435-448.

Ferguson, C. J., Coulson, M. y Barnett, J. (2011). A meta-analysis of pathological gaming prevalence and comorbidity with mental health, academic and social problems. Journal of Psychiatric Research, 45, 1573-1578. doi:10.1016/j.jpsychires.2011.09.005.

Fernández-Villa, T., Alguacil, J., Almaraz, A., Cancela, J.M., Delgado-Rodríguez, M., García-Martín, M., ... Martín, V. (2015). Uso problemático de internet en estudiantes universitarios:factores asociados y diferencias de género. Adicciones, 27, 265 - 275.

Grant, J. E., Potenza, M. N., Weinstein, A. y Gorelick, D. (2010). Introduction to Behavioral Addictions. The American Journal of Drug and Alcohol Abuse, 36, 233-241. doi:1 0.3109/00952990.2010.491884.

Griffiths, M. D. y Meredith, A. (2009). Videogame Addiction and its Treatment. Journal of Contemporary Psychotherapy, 39, 247-253. doi:10.1007/s10879-009-9118-4.

Holtz, P. y Appel, M. (2011). Internet use and video gaming predict problem behavior in early adolescence. Journal of Adolescence, 34, 49-58. doi:10.1016/j.adolescence.2010.02.004.

Kaufman, J., Birmaher, B., Brent, D., Rao, U., Flynn, C., Moreci, P., ... Ryan, N. (1997). Schedule for Affective Disorders and Schizophrenia for school-age children present and lifetime version (K-SADS-PL): Initial reliability and validity data. Journal of the American Academy of Child E् Adolescent Psychiatry, 36, 980-988.

King, D. L. y Delfabbro, P. H. (2014). Internet gaming disorder treatment: a review of definitions of diagnosis and treatment outcome. Journal of Clinical Psychology, 70, 942-955. doi:10.1002/jclp.22097.

Király, O., Nagygyörgy, K., Griffiths, M. D. y Demetrovics, Z. (2014). Problematic Online Gaming. Behavio- 
ral Addictions: Criteria, Evidence, and Treatment,4 61-97. doi:10.1016/B978-0-12-407724-9.00004-5.

Ko, C. H., Yen, J. Y., Chen, C. C., Chen, S. H. y Yen, C. F. (2012). The association between Internet addiction and psychiatric disorder: A review of the literature. European Psychiatry, 27, 1-8. doi:10.1016/j.eurpsy.2010.04.011.

Kuss, D. J., Van Rooij, A. J., Shorter, G. W., Griffiths, M. D. y Van de Mheen, D. (2013). Internet addiction in adolescents: Prevalence and risk factors. Computers in Human Behavior, 29, 1987-1996. doi:10.1016/j.chb.2013.04.002.

Lemos, I. L., De Abreu, C. N. y Sougey, E. B. (2014). Internet and video game addictions: a cognitive behavioral approach. Revista de Psiquiatria Clínica, 41, 82-88. doi:10.1590/0101-60830000000016.

Lopez-Fernandez, O., Honrubia-Serrano, M. L., Baguley, T.y Griffiths, M. D. (2014). Pathological video game playing in Spanish and British adolescents: Towards the exploration of Internet Gaming Disorder symptomatology. Computers in Human Behavior, 41, 304-312. doi:10.1016/j.chb.2014.10.011.

Marco, C. y Chóliz, M. (2014). Tratamiento cognitivo-conductual de la adicción a videojuegos de rol online: fundamentos de propuesta de tratamiento y estudio de caso Introducción. Anales de Psicología, 30, 46-55.

Matalí-Costa, J., Serrano-Troncoso, E., Pardo, M., Villar, F. y San, L. (2014). Behavior Social Isolation and the "Sheltered" Profile in Adolescents with Internet Addiction. Journal of Child E् Adolescent Behavior, 2, 139. doi:10.4172/jcalb.1000139.

Petry, N. M. y O'Brien, C. P. (2013). Internet gaming disorder and the DSM-5. Addiction, 108, 1186-1187. doi:10.1111/add.12162.

Petry, N. M., Rehbein, F., Gentile, D.A., Lemmens, J. S., Rumpf, H. J., Mößle, T., Bischof, G., ... O’Brien, C.P. (2014). An international consensus for assessing internet gaming disorder using the new DSM-5 approach. Addiction, 109, 1399-1406. doi:10.1111/add.12457.

Rehbein, F., Kliem, S., Baier, D., Mößle, T. y Petry, N. M. (2015). Prevalence of internet gaming disorder in German adolescents: diagnostic contribution of the nine DSM-5 criteria in a state-wide representative sample. $A d$ diction, 110, 842-851. doi:10.1111/add.12849.

Teo, A. R. (2013) . Social isolation associated with depression: A case report of hikikomori. International Journal of Social Psychiatry, 59, 339-341. doi:10.1177/0020764012437128.

Ulloa, R. E., Ortiz, S., Higuera, F., Nogales, I., Fresán, A., Apiquian, R., ... de La Peña, F. (2006). Interrater reliability of the Spanish version of Schedule for Affective Disorders and Schizophrenia for School-Age Children-Present and Lifetime version (K-SADS-PL). Actas Españolas de Psiquiatria, 34, 36-40. doi:51110615 [pii].

Vallejos, M. y Capa, W. (2010). Video Juegos: Adicción y Factores Predictores. Avances en Psicología, 18, 103-110.
Van Rooij, A. J., Kuss, D. J., Griffiths, M. D., Shorter, G. W., Schoenmakers, M. T., y Van de Mheen, D. (2014). The (co-) occurrence of problematic video gaming, substance use, and psychosocial problems in adolescents. Journal of Behavioral Addictions, 3, 157-165. doi:10.1556/ JBA.3.2014.013.

Yau, Y. y Potenza, M. (2014). Internet Gaming Disorder. Psychiatric Annals, 44, 379-383. doi:10.3928/0048571320140806-05.

Yen, J.Y., Ko, C.H., Yen, C.F., Wu, H.Y. y Yang, M.J. (2007). The comorbid psychiatric symptoms of Internet addiction: attention deficit and hyperactivity disorder (ADHD), depression, social phobia, and hostility. The Journal of Adolescent Health, 41, 93-98. doi:10.1016/j.jadohealth.2007.02.002.

Young, K. S. (2007). Cognitive behavior therapy with Internet addicts: treatment outcomes and implications. Cyberpsychology $\mathcal{E}$ Behavior: The Impact of the Internet, Multimedia and Virtual Reality on Behavior and Society, 10, 671679. doi:10.1089/cpb.2007.9971. 